\title{
Balkanologie
}

Balkanologie Revue d'études pluridisciplinaires

Vol. II, nº 1 | 1998

Volume II Numéro 1

\section{Mythes, symboles, rituels. Le pouvoir historique des « signes » en Europe du Sud-Est aux XIX et XX siècles, colloque, Bonn, 15-17 septembre 1997}

\section{Milan Kosanović}

Traducteur : Suzana Đurić

\section{(2) OpenEdition}

\section{Journals}

Édition électronique

URL : http://journals.openedition.org/balkanologie/246

DOI : 10.4000/balkanologie.246

ISSN : 1965-0582

\section{Éditeur}

Association française d'études sur les Balkans (Afebalk)

\section{Édition imprimée}

Date de publication : 1 juillet 1998

ISSN : 1279-7952

\section{Référence électronique}

Milan Kosanović, « Mythes, symboles, rituels. Le pouvoir historique des « signes » en Europe du SudEst aux XIX et XXe siècles, colloque, Bonn, 15-17 septembre 1997 », Balkanologie [En ligne], Vol. II, n 1 । 1998, mis en ligne le 02 juin 2008, consulté le 17 décembre 2020. URL : http://

journals.openedition.org/balkanologie/246 ; DOI : https://doi.org/10.4000/balkanologie.246

Ce document a été généré automatiquement le 17 décembre 2020.

(c) Tous droits réservés 


\title{
Mythes, symboles, rituels. Le pouvoir historique des « signes » en Europe du Sud-Est aux XIX et XX siècles, colloque, Bonn, 15-17 septembre 1997
}

\author{
Milan Kosanović \\ Traduction : Suzana Đurić
}

\section{NOTE DE L'ÉDITEUR}

Texte traduit du serbo-croate par Suzana Đurić.

1 L'Europe du sud-est jouit depuis plusieurs années de l'attention toute particulière des sciences sociales. Hélas, cet intérêt est également provoqué par les événements qui se sont déroulés ces dernières années dans l'ancienne Yougoslavie. Une partie des recherches sur l'Europe du sud-est concerne les débats et les effets des mythes parmi les nations de cette partie de l'Europe. Le colloque de Bonn devait précisément contribuer à la recherche sur les courants sociaux en Europe du sud-est. Organisé par les professeurs Wilfried Potthoff de la Chaire de slavistique et Dittmar Dahlmann de la Chaire d'histoire de l'Europe du sud-est à l'université de Bonn, ce colloque avait un caractère interdisciplinaire. Au total, 17 contributions dans les domaines de la slavistique, de l'histoire contemporaine, de la sociologie, de l'ethnologie, de l'histoire de l'art, romane et classique, divisées en quatre sections sur une période de trois jours ont pu être entendues.

2 Le professeur Hans-Joachim Gehrke, de l'université de Freibourg, a présenté un rapport d'introduction, intitulé «Mythes, histoire et identité collective. Antike exempla et leurs effets tardifs». Il est intervenu à propos des rapports envers l'histoire durant 
l'Antiquité. Il n'existait alors pas de frontière évidente entre histoire et poésie. Les Histoires d'Hérodote, ou encore La guerre du Péloponnèse de Thucydides, ont eu à peu près les mêmes effets sur la création d'une conscience historique collective que les tragédies d'Eschyles, d'Euridipe ou de Sophocle.

3 Gehrke a introduit le concept d'histoire intentionnelle au moyen duquel il a expliqué que le devoir de l'histoire antique était d'intégrer à la conscience historique collective d'un groupe ethnique certains buts ethniques ou nationaux. Les guerres persiques et les victoires athéniennes ne sont, dans ce contexte, que la suite logique d'une histoire glorieuse au cours de laquelle les Athéniens avaient déjà triomphé des titans, des amazones et des géants. Les victoires sur les Perses n'étaient que la fin provisoire d'une histoire monumentale. L'histoire a, généralement, été écrite a posteriori, à un présent constamment adapté. De par sa fonction, elle a servi le présent. Celle d'un mythe également très répandu parmi les Hellènes fut même de faire la différence entre Hellènes et Barbares. Au sens premier, les Barbares étaient non-Hellènes, c'est-à-dire des peuples dont le panthéon divin était différent de celui des Hellènes, des peuples qui ne parlaient pas grec et qui n'appartenaient à aucun des trois peuples mythologiques helléniques, les Ioniens, les Doriens et les Éoliens. Plus tard, c'est de cette manière que l'on marquait la différence entre liberté et despotisme, entre culture grecque et primitivisme des autres. Ce sens figuré s'est, pour ainsi dire, maintenu chez tous les peuples d'Europe que les Grecs de l'Antiquité classaient parmi les Barbares, tandis que cette idée, dans les langues européennes modernes, est utilisée, sous forme de connotation et de ressentiments déterminés, pour désigner, entre autres, les Grecs euxmêmes.

\section{La production des mythes modernes et le romantisme politique}

4 La première session débuta par l'intervention du professeur d'ethnologie Vitomir Belaj de l'université de Zagreb, "Le mythe des "pré-Croates" et ses caractéristiques ». Il a passé en revue les différentes théories mythologiques sur l'origine des Croates, sans la moindre ironie, sous l'œil amusé de l'auditoire. Il a restreint les thèses, d'un nombre considérable, à trois groupes :

5 (i) les Croates tirent leur origine des Avars. Selon cette thèse, il n'y avait pas de Croates avant le VII ${ }^{\mathrm{èm} e}$ siècle, seulement des Avars. L'ethnonyme "croate" proviendrait d'un titre avar ;

(ii) les Croates tirent leur origine des peuples du Caucase. Il existe des étymologies linguistiques comportant le terme de croate, mais aussi de polonais, de tchèque et de serbe ;

(iii) leur origine serait perse. Une des versions propose comme argument des étymologies linguistiques de certains noms géographiques de l'Iran actuel (la région d'Horawati). Il est toutefois également possible de trouver ce genre de similitude avec des toponymes d'Asie centrale, d'Afghanistan, d'Inde, ... La seconde version a vu le jour à la seule fin de distinguer les Croates des Serbes. Les Croates tirent leur origine des Perses, tandis que les Serbes doivent la leur aux esclaves romains originaires d'Afrique, la couleur sombre de leur peau et leurs cheveux frisés en feraient d'ailleurs foi. L'origine croate serait extraite de documents persans du XIV ème siècle avant JC, dans lesquels, évidemment, on parlait de Croates. Ils auraient ensuite quitté leur terre natale 
et colonisé toute l'Europe de l'est et du sud-est jusqu'à l'Oural. Si bien que, selon cette thèse, les Slaves tireraient leur origine des Croates.

Belaj a mentionné que ce genre d'argumentations mythologiques était d'une actualité récente. Elles ne servent pas seulement d'explication à l'origine des Croates, mais également d'argument à l'impossibilité d'une cohabitation avec les Serbes dans un même pays, sans parler d'une quelconque fraternité !

7 Le dr. Wolfgang Kessler de l'université de Herne est intervenu en second, avec un exposé intitulé « Des castes à la notion d'État : l'idéologie de classe et la découverte de l'histoire médiévale dans l'idéologie nationale croate ». Kessler rappela que l'illyrisme est une idée pré-yougoslave, provenant principalement du milieu bourgeois croate, que les Serbes avaient également adopté. Il a mentionné que l'union entre Croates et Serbes était beaucoup plus forte avant 1860. Le patriarche serbe Vlačić avait même intronisé le Ban croate Jelačić. Toutefois, entre 1860 et 1870 , l'idée de l'illyrisme a perdu de son intensité et de son sens avec l'apparition d'un mouvement national croate fort qui a été provoqué, en partie, par le fait que l'élite politique serbe de l'époque était devenue le principal vecteur de l'idée yougoslave.

Le préambule des constitutions de l'État croate moderne repose, en grande partie, sur les postulats du mouvement national croate de l'époque. Il est également possible d'établir des parallèles entre la période s'étalant de 1860 à 1916 et le problème de la Krajina.

9 Le professeur de slavistique Wilfried Potthoff de l'université de Bonn s'est penché sur «L'histoire et les mythes historiques dans la littérature yougoslave ». Il a commencé avec la présentation de l'un des mythes qui eut le plus de conséquences sur l'histoire des peuples yougoslaves, le mythe illyrien.

Potthoff a rattaché les débuts du mouvement illyrien à Vinko Pribojević au XVİ̀me siècle, les Illyriens représentants alors les ancêtres des Slaves. Puisque l'on classait les Macédoniens et les Romains parmi les Illyriens, les rois de Macédoine, Aristote, ainsi que les empereurs romains étaient, par conséquent, d'origine slave. À peu près au même moment à Dubrovnik, on commençait a éditer des recueils de blasons dans lesquels les symboles les plus récurrents étaient le croissant de lune et l'étoile. Ces symboles ont, cependant, des racines beaucoup plus anciennes: on pouvait déjà les trouver gravés sur les monuments funéraires en Bosnie. Ils ont ensuite, par le biais des recueils d'armoirerie de Dubrovnik, trouvé leur place sur le blason, plus tardif, de l'Illyrie et sont ainsi devenus les principaux symboles du mouvement illyrien.

11 Le manque de sources historiques sur lesquelles on pourrait se fonder est un problème crucial. L'œuvre de Mavro Orbinj, Le royaume des Slaves, représente ainsi l'unique source en ce qui concerne certaines périodes de l'histoire médiévale serbe, telle que la bataille de Kosovo par exemple. Les possibilités de vérification sont de ce fait des plus limitées. L'œuvre d'Orbinj a même servi de source au mouvement illyrien. Potthoff a également mentionné la première tentative de création d'une langue illyrienne par Juraj Križanić. Plus tard, aux environs de 1700, Vitezović a unifié l'Illyrie et la Croatie et, ce faisant, intégré dans une seule et même Croatie, la Dalmatie, la Slavonie, la Croatie intérieure (Croatia uterior), la Krajina, la Bosnie, l'Herzégovine et la Serbie. Les jésuites, dont l'ordre portait même le nom de Collegium Illyricum en Bosnie et en Croatie, jouèrent un rôle particulièrement déterminant en ce qui concerne le développement du mythe 
illyrien. Le premier résultat politique de plusieurs siècles de création et d'entretien du mythe illyrien a été la fondation par Napoléon de la province illyrienne en 1809.

Le mythe illyrien ne se limitait pas aux écrivains, poètes et chroniqueurs croates : s'y sont joints, plus tard, les intellectuels slovènes et serbes. Le slovène Valeri Vodnik, au début $\mathrm{du}$ XIX̀̀me siècle, a écrit une ode à l'Illyrie dans laquelle Napoléon dit «Illyrie, lève-toi!». En Serbie, en 1844, bien que sous un autre nom, le Načertanije d'Ilija Garašanin, dans lequel il demande la re-création de l'empire de Dušan, a contribué au développement de l'idée du mouvement illyrien. Chez les Serbes, cependant, le mythe du Kosovo a été d'une importance bien plus forte, et ce jusqu'à notre époque. À ce sujet, Potthoff a fait mention du memorandum de l'Académie serbe des sciences et des arts et de son vice-président, Antonije Isaković, qui a déclaré que les Serbes se souvenaient, à chaque fois qu'ils étaient dans une situation difficile, du Kosovo, de Karađorđe et des chants populaires épiques. Pour conclure, il a cité quelques rares critiques du mythe du Kosovo.

13 Helmut Keipert, professeur de slavistique de l'université de Bonn, a évoqué la première codification de la grammaire serbe par Vuk Karadžić en 1814, intitulée Pismenici serbskog jezika (Grammaire de la langue serbe). Il s'est intéressé à la préface dans laquelle Karadžić décrit les principes auxquels il s'est tenu lors de l'élaboration de la Grammaire. Keipert a extrait :

(i) mettant l'accent sur le concept populaire de l'époque de "langue maternelle", Karadžić tente de présenter son œuvre comme un devoir patriotique que les autres ont délaissé ;

(ii) il relève avec modestie qu'il ne possède qu'une qualification limitée de codification ;

(iii) Karadžić souligne clairement la simplicité de sa codification :

- elle n'est même pas proche des codifications de l'allemand Adelung et du tchèque Dobrovski, ce qui n'est d'ailleurs pas son intention ;

- elle n'englobe qu'une seule partie : la syntaxe fait défaut ;

- son rôle n'est que d'encourager les autres, ceux qui sont plus qualifiés ;

- en orthographe, un principe simple est employé : «écris comme tu parles et lis comme c'est écrit »;

- il s'agit d'une description de cette langue serbe parlée "loin des villes".

15 Keipert a voulu montrer, au moyen de son exposé, que la Grammaire de la langue serbe présente une répétition des arguments que Jernej Kopitar, linguiste slovène, avait déjà mentionné dans son idée de standardisation des langues slovène, grecque et serbe avant même d'avoir fait la connaissance de Vuk Karadžić. Kopitar a eu une influence décisive sur la création de la codification serbe, non seulement par le biais de ses critiques, mais également par celui de son influence sur d'autres critiques (tels que J. Grimm, J. S. Vater). Cela a permis la naissance d'une image positive de Karadžić qui a contribué, de manière considérable, à la naissance de la "Légende de Vuk".

Le dr. Katrin Boekh, collaboratrice scientifique de l'Institut de l'Europe de l'est de Münich, a clos la première session avec son exposé sur «Le mythe de l'étranger dans les Balkans ». La problématique était qu'il existe parmi les Slaves du sud une croyance mythologique en une conspiration des États étrangers à leur encontre. Son analyse concernait surtout les Serbes. Elle a toutefois précisé que son hypothèse était également valable pour les autres nations de l'Europe du sud-est. D'après elle, les Serbes considèrent les Turcs, les Albanais, les Allemands ou l'Occident en général, ainsi que Rome, c'est-à-dire le Pape, comme étant les complices d'un grand complot contre le 
peuple serbe. Elle a souligné que la différence fondamentale entre les Serbes et ces nations était la religion.

L'orthodoxie, qui n'a pas connu le processus des réformes, est porteuse du développement culturel. La disposition au dialogue faisant défaut, il est, par conséquent, possible de manipuler plus aisément une société orthodoxe fermée sur elle-même qu'une autre ouverte sur le monde. Elle en chercha la raison dans le manque de civilisation des peuples balkaniques, dont les sociétés sont, de façon prédominante, rurales. Les Serbes considèrent être une nation divine ayant connu le génocide et l'exode, ils mènent une guerre sainte, ... Les tombes deviennent des symboles nationaux. Leurs souffrances sont apparentées à celles du Christ. Dans ce contexte, Boekh fit mention de l'iconologie des politiciens contemporains dont les photographies sont portées dans les manifestations. À cette occasion, elle cita le $600^{\text {ème }}$ anniversaire de la bataille du Kosovo en 1989, où l'on pouvait voir quantité de photographies de Milošević, ainsi que diverses manifestations à travers la Bosnie-Herzégovine où l'on exhibait celles de Radovan Karadžić et de Biljana Plavšić.

Le revers de ce mythe de l'antipathie envers l'extérieur est la "mythologisation" du concept d'“Europe". Les Serbes considèrent leur Église orthodoxe comme l'héritière de l'Église byzantine, par laquelle ils trouvent leur place au sein de l'Europe. La période du pouvoir osmanli a été vécue, en Serbie, comme un état de souffrance et de torture permanent pendant 500 ans. Boekh estime que les effets de l'histoire sont encore actuellement prégnants chez les Serbes. Elle légitima cette hypothèse par l'incompréhension des Serbes vis-à-vis des sanctions qu'on leur a imposé, ainsi que le mythe d'une guerre juste menée en Croatie et en Bosnie-Herzégovine. Elle a conclu son exposé par l'affirmation que le peuple lui-même crée son propre mythe.

Critiquant son intervention, Suppan a déclaré que les Serbes étaient un peuple sécularisé sur lequel Boekh avait surestimé l'influence de la religion. Les inexactitudes historiques, nombreuses dans l'exposé, ont également été critiquées. On a notamment souligné que l'Église orthodoxe serbe n'était pas globalement contre l'Occident, mais qu'on y trouvait, tout comme dans d'autres organisations ecclésiastiques, divers courants.

\section{Idées étatiques et mythes nationaux}

20 Johannes Burkhardt, professeur d'histoire moderne à Augsburg, a souligné dans sa contribution, "Les mythes nationaux et la naissance des guerres", que les mythes pouvaient, dans certaines conditions, provoquer des guerres. Il a ouvert son exposé avec l'hypothèse que le mythe du Kosovo était l'un des instigateurs des guerres yougoslaves contemporaines. À cette occasion, il a laissé échapper une "erreur" historique : l'affirmation que la guerre de Slovénie avait débuté le jour de la Saint-Guy (Vidovdan), tout comme la bataille du Kosovo. Cette hypothèse appartient à un mythe du mythe du Kosovo circulant parmi les historiens et slavistes allemands. L'essence de ce mythe est que l'attentat contre l'héritier du trône d'Autriche, Franz-Ferdinand, eut lieu le 28 juin 1914, vidovdan. Les défenseurs de ce mythe rattachent également le début de la guerre en Slovénie au jour du vidovdan, le 28 juin 1991. Partant du principe que les dates ne sont pas choisies au hasard, on peut en tirer la conclusion que les Première Guerre mondiale et yougoslave ont été provoquées par le mythe du Kosovo. Ainsi, on oublie, plus ou moins sciemment, que la guerre en Slovénie a débuté le 25 juin et que 
cette date avait été choisie par les Assemblées des Républiques de Slovénie et de Croatie. L'exposé n'a, cependant, pas souffert de cette erreur "mythologique", car Burkhardt travaille sur l'histoire moderne dans les pays germaniques; il a donc centré son exposé sur cette période, c'est-à-dire sur la Guerre de Trente Ans entre protestants et catholiques qui avait, elle, débuté à cause de la célébration d'un centenaire: la proclamation des thèses de Luther.

21 Le professeur Sergio Bonazza, de l'université de Verone, évoqua «Le mythe habsbourgeois chez les Slaves du sud». Il émit l'hypothèse que le mythe de la monarchie habsbourgeoise chez les Slaves du sud renfermait en soi plusieurs mythes. Le premier est celui d'Austria felix, ensuite viennent les mythes d'un État multinational heureux et du monarque paternaliste. Ces mythes ont été créés au XIX ${ }^{\mathrm{eme}}$ siècle dans le but concret de renforcer la monarchie habsbourgeoise. La problématique de l'intervention de Bonazza était d'établir la manière dont s'était développé le mythe habsbourgeois parmi les Slaves du sud d'Autriche-Hongrie.

Partant du principe que la littérature est le produit de la culture d'un peuple, qu'à une période elle reflète de la meilleure manière qui soit les valeurs historiques de ce peuple, Bonazza a articulé son argumentation au moyen d'exemples littéraires. Il démontra que ce mythe s'était particulièrement développé chez les Slovènes (France Prešern) et dans une moindre mesure chez les Croates (Ivan Mažunarić), qui, avec l'illyrisme, ont eu une autre alternative, tandis qu'il n'est présent chez les Serbes qu'en tant que phénomène mineur.

23 Au cours de la discussion, Keipert a mentionné qu'il fallait bien réfléchir à l'essence de cette hypothèse, car des phénomènes semblables pouvaient être suivis dans pratiquement toute l'Europe à l'époque. Suppan et Steinke se sont joints à cet avis.

La seconde intervention a été faite par le professeur Peter Bartl, de l'université de Münich, qui a parlé du «mythe historique chez les Albanais». Le réveil national albanais, connu sous le nom de Rilindja (renouveau), est arrivé tardivement, dans la seconde moitié du XIX ${ }^{\text {ème }}$ siècle seulement. Les peuples voisins n'ont pas été les seuls à s'opposer à la création d'un État national albanais, mais également les Albanais de confession musulmane qui désiraient demeurer dans l'État ottoman. C'est pour cette raison que l'intelligentsia albanaise, puisqu'il n'y avait pas de tradition historique médiévale, a tenté de construire un mythe historique sur l'origine des Albanais.

L'essence de ce mythe est que les Albanais sont un peuple très ancien, si ce n'est le plus ancien, des Balkans, dont ses représentants ont toujours vécu dans la région où ils se trouvent actuellement. Il n'y a jamais eu d'interruption dans la continuité de l'histoire albanaise, car les occupations étrangères n'étaient pas en mesure d'interrompre la tradition nationale. Bartl a cité trois publications dans lesquelles s'est développé ce mythe au XIX ${ }^{\text {ème }}$ siècle. Ce sont Discorso sull'origine, costumi, e stato attuale della nazione albanese (Débats sur l'origine, les coutumes et l'état actuel de la nation albanaise) d'Angelo Masci (Naples, 1807), Albanien und die Albanesen. Eine historisch-kritische Studie, von Wassa Effendi, Beamter der Kaiserlich Türkischen Regierung, Christlich-Albanischer Nationalität (L'Albanie et les Albanais. Une étude historico-critique de Vasa Effendi, fonctionnaire du pouvoir impérial turc, de nationalité albano-chrétienne), (Berlin, 1879) et le programme du mouvement national albanais, Was war Albanien, was ist es und was wird es werden? Gedanken und Betrachtungen über die unser geheiligtes Vaterland Albanien bedrohenden Gefahren und deren Abwendung (Ce que fut l'Albanie, ce qu'elle est et ce qu'elle sera. Réflexions et discussions sur les dangers terribles qui menacent notre 
sainte patrie l'Albanie et leur rejet) de Sami Frashëri (Bucarest, 1889). Masci, qui était d'origine italo-albanaise et haut-fonctionnaire de la ville de Naples à l'époque de Napoléon, décrit les Albanais comme les héritiers des Illyriens et des Macédoniens, qu'il considère partager la même origine. Selon cette idée, les Albanais parlent macédonien. Depuis la conquête romaine, les Albanais n'ont plus agit sur l'histoire, mais l'ont seulement subie. Skender Beg fut l'unique exception.

La discussion de Vasa Effendi débute avec les Peslages, auxquels il attribue le droit de primauté dans les Balkans, comme peuple européen le plus ancien. Les Illyriens, les Épirotes et les Macédoniens étaient des Peslages. En tant que fonctionnaire du gouvernement ottoman, sa description en est globalement positive, et il regrette que les Albanais, durant la période du Tanzimat (milieu du XIX ${ }^{\text {ème }}$ siècle), ne se soient pas joints au mouvement de modernisation de l'empire ottoman. Dans son œuvre, il demandait que tous les territoires habités par des Albanais soient réunis en un seul et même villayet. Vasa Effendi, dont le nom de baptême est Paško Vasai, et qui n'a pas changé de religion, a, comme haut fonctionnaire de l'État ottoman, accédé au titre de pacha et été nommé gouverneur au Liban. Sami Frashëri s'est, en grande partie, basé sur l'œuvre de Vasa. Il trouve l'origine du mot arban dans le terme albanais utilisé pour champ, concluant que les Albanais étaient à l'origine des paysans-agriculteurs. Durant la période pré-antique, ils ont peuplé non seulement les Balkans, mais également l'Asie mineure et l'Italie. Les Étrusques tirent précisément leur origine de ces premiers colonisateurs de l'Italie. Les Hellènes et les Macédoniens également, si bien qu'Alexandre le Grand, ainsi que son père Philippe, étaient aussi albanais. Avec l'arrivée des Slaves dans les Balkans, les Albanais qui vivaient en Thrace et en Macédoine ont dû quitter leurs terres, tandis que les Albanais de Bosnie, de Dalmatie et du Monténégro se sont mêlés avec les Slaves et ont adopté leur langue.

27 Frashëri, tout comme Vasa, considère le pouvoir ottoman d'un œil plutôt positif, et souligne la continuité albanaise. Il considère que les Albanais ont toujours travaillé pour les autres (Sinan pacha et Mehmet Ali pacha) et c'est la raison pour laquelle l'histoire albanaise a connu un développement défaillant. Le seul qui ait œuvré pour l'Albanie est Skender Beg. Après la Seconde Guerre mondiale, la toute jeune historiographie albanaise s'est, d'une manière générale, approprié les thèses des temps de la Rilindja.

28 À la question de savoir dans quelle mesure la naissance du mythe albanais a pu servir les intérêts de l'Autriche-Hongrie, Bartl a répondu qu'immédiatement après la publication de l'œuvre de Frashëri, on a émis l'hypothèse qu'il s'agissait d'un plagiat des services d'informations autrichiens. Il était d'ailleurs de notoriété publique que Frashëri lui-même niait être l'auteur de l'étude mentionné.

\section{La transformation des mythes en symboles et rituels}

Le professeur d'histoire de l'art de l'université de Bonn, Heinrich-Josef Klein, a consacré son exposé aux «Célébrations du millénaire en Hongrie en 1896 ». On commémorait alors le millénaire de la colonisation hongroise mythologique de la plaine panonienne. Pour cette occasion, la ville de Budapest avait connu une importante réorganisation urbaine. Diverses représentations furent construites, telles que le Parlement, la cour de cassation, la banque nationale et le musée d'arts appliqués, ainsi que la route périphérique à la capitale (Andrássy-út) avec la Place des Héros. Dans le cadre des 
célébrations, on a organisé une grande exposition, qui a reçu près d'un million de personnes par mois et cinq millions vers la fin de l'exposition, ainsi que des excursions dans toute la Hongrie. Au centre de la célébration se tenait la Couronne de St. Stefan, symbole de l'unité hongroise. La Couronne, gardée au palais de Buda, a été solennellement transportée au Parlement, ouvrant ainsi la séance plénière, puis remportée au palais de Buda dans une procession tout aussi solennelle.

Une centaine d'années plus tard, en 1996, on célébrait le "millecentenaire", et, à cette occasion, dans des conditions politiques complètement différentes, on a fêté le même jubilé que cent ans auparavant, avec un programme beaucoup plus modeste. Une fois de plus, la procession du transfert de la Couronne du palais jusqu'au Parlement et son retour occupait le centre de la cérémonie. Parmi les premiers suivants de la Couronne se trouvait l'ancien Président des États-Unis d'Amérique, Jimmy Carter, qui, du temps de son mandat, avait restitué à la Hongrie sa Couronne perdue : lors de la Seconde Guerre mondiale, elle avait été sortie du pays et retrouvée plus tard aux États-Unis. Un film a même été tourné sur la conquête mythologique de la plaine panonienne par Arpad (l'acteur italien Franco Nero ayant tenu le rôle principal). Des concerts, des expositions, des représentations théâtrales, etc., ont été organisés.

Klein a présenté des vieilles photographies du millénaire, ainsi que des extraits du film sur la conquête d'Arpad et des enregistrements vidéo tournés lors de différentes manifestations pour le "millecentenaire".

Peter Hasslinger, collaborateur scientifique à l'université de Freibourg, est intervenu ensuite «À propos de la continuité et des ruptures: l'image de l'Autriche dans la caricature hongroise de 1895 à 1990 ». Il a tenté de montrer l'influence des caricatures sur la naissance, le développement et l'action des mythes politiques. Le point central de l'exposé était constitué de caricatures de la période s'étendant de 1895 à 1914, dans lesquelles l'image de l'Autriche et des Autrichiens était soumise à une certaine transformation. Au début de cette période, les Autrichiens sont présentés comme les exploiteurs des Hongrois. À partir de 1900, l'image change et on montre les Autrichiens pauvres et misérables, tandis que les Hongrois sont représentés en meilleure santé, plus riches et plus intelligents que leurs voisins. Hasslinger a souligné que malgré les caricatures présentées tirées de différents magazines, l'image de l'Autriche ne correspond pas à la réalité. Elle est soumise aux changements quotidiens qui sont le produit d'un processus politique. Il a établi que l'image de l'Autriche était hétérostéréotypée, tandis que celle des Hongrois était autostéréotypée et il en a tiré la conclusion que la représentation du potentiel de concurrence et de conflit nuit à l'autre en général.

Le professeur de slavistique Klaus Steinke de l'université d'Erlangen s'est penché sur la typologie des mouvements de renaissance nationale en Europe du sud-est dans son intervention «Le renouveau bulgare et Scoala ardealeana». Il a comparé le développement des renouveaux bulgare et roumain qui ont débuté dans la seconde moitié du XVIII ${ }^{e ̀ m e}$ siècle, comparaison inhabituelle, car il n'existe aucun lien direct entre les deux. Steinke, cependant, soutint qu'il existe nombre de similitudes typologiques permettant une telle comparaison. Il s'est, toutefois, limité à deux aspects, le peuple et la langue.

34 La langue est une composante essentielle dans les deux processus. Les personnalitésclefs sont, pour les Bulgares, Pajsije Hilandarski avec son œuvre Histoire slavo-bulgare et, pour les Roumains, Gheorghe Sincai et Patru Maior. Une importance particulière est à 
attribuer aux auteurs des premières grammaires et abécédaires. Chez les Bulgares, ce sont Peter Beron et Neofit Rilski ; chez les Roumains, G. Sincai, à nouveau, et Samuel Klein-Micu.

À la différence du cas bulgare où il existait une forte tradition médiévale, il n'existait en Roumanie, pour ainsi dire, aucune tradition. Le cas roumain était tellement méconnu qu'encore au début du XIX ${ }^{\text {ème }}$ siècle, les romanistes occidentaux ne connaissaient même pas la langue roumaine. L'origine des Roumains était mythologique, deux courants se distinguant : a) les Roumains tirent leur origine des Daces et 2) ils ont pour ancêtres les Romains. Dimitrije Kantemir défendait la seconde thèse, tandis que la première fut plus répandue dans des périodes plus tardives, surtout après la Seconde Guerre mondiale. Selon cette hypothèse, l'origine de la langue latine est dace. Au XIX ${ }^{\text {ème }}$ siècle, l'“École transylvaine" fut d'une grande importance : ses membres ont cherché les racines de la langue roumaine dans la langue latine et ont ainsi modifié l'orthographe des mots d'origine slave afin de les rapprocher le plus possible du latin. Ainsi, le mot slave razboi (guerre) s'écrivait res belli. Steinke s'est, en tout cas, basé sur le travail du scientifique moderne roumain Lucian Boiae, dont l'œuvre a été éditée en 1997.

Le professeur d'histoire d'Europe du sud-est, Arnold Suppan de l'université de Vienne, est intervenu sur «La caricature comme source des mythes modernes. Comparaison entre Serbes, Croates et Slovènes ». Il a étudié l'image qu'ils avaient de l'Autriche et des Autrichiens durant la période de 1885 à 1995. La plupart des caricatures en question proviennent de Slovénie et de Croatie.

37 Suppan a situé le concept de caricature et son importance pour la science historique. Ainsi, il a déclaré que la caricature est importante du fait que ce que l'on désire montrer se trouve restreint aux symboles les plus significatifs et qu'une fois son caractère comique soustrait, il ne reste que cette représentation "sérieuse" d'un thème concret. Le but des caricatures est de montrer au public des phénomènes sociaux et politiques, des relations, des processus, etc., d'une manière particulièrement simplifiée. Suppan a souligné que lorsque la caricature est prise comme une source historique, on se doit de prendre en compte son caractère particulier ainsi que son intention spécifique. Dans le cas contraire, l'utilité de la caricature en tant que source historique serait négative, offrant une image erronée et exagérée d'un thème déterminé.

Suppan a présenté diverses caricatures et fait une démonstration de la dynamique de l'image de l'Autriche et des Autrichiens dans trois parties différentes de l'ancienne Yougoslavie à diverses périodes.

Le dernier exposé de la troisième section a été présenté par Dorothea Schell, collaboratrice scientifique du séminaire d'ethnologie de l'université de Bonn. Sa contribution, «Perspectives ethnologiques de recherches sur les symboles et les rituels. Exemples recueillis dans la périphérie grecque", était basée sur des recherches empiriques menées en Grèce dans la période 1990-1995, notamment sur l'île de Samos. La problématique de son travail consistait à déterminer dans quelle mesure les mythes pouvaient servir à l'unification des communautés nationales dans leurs actions politiques.

Comme exemple, elle a cité la fonction de l'hymne et du drapeau en tant que symboles nationaux visant à créer un sentiment national collectif. Elle donne la même signification aux monuments à connotation politico-nationale. Ses recherches étaient centrées sur les événements les plus récents en Grèce, liés à la naissance de la République ex-yougoslave de Macédoine et au développement du sentiment politique 
du nous parmi les Grecs. C'est dans cette intention même que certains symboles historiques ont obtenu une caractéristique nationale. Le plus significatif de ces symboles est l'étoile de la Vierge, devenue le principal symbole du droit exclusif de la Grèce sur le nom de Macédoine. Elle a été retrouvée sur une tombe dans la partie grecque de la Macédoine, que l'on croit être celle du père d'Alexandre le Grand, Philippe II, le Roi de Macédoine. Du point de vue archéologique cependant, il n'a pas été prouvé qu'il s'agissait bien de la tombe de Philippe II et certains affirment qu'il s'agirait de celle de Philippe V.

41 Le symbole a acquis une grande popularité et est devenu non seulement un symbole politique, mais également un symbole de mode et de style des années 1990 en Grèce. Les services de porcelaine, les bijoux et autres objets décoratifs sont ornés de l'étoile de la Vierge, les factures de téléphone, d'électricité et d'eau portent au dos la marque de ce symbole. Schell, cependant, ne considère pas qu'il s'agisse d'un mythe exclusivement construit d'en haut, mais qu'il s'est développé de lui-même avec l'aide de "dilettantes bien intentionnés qui se sont efforcés de participer aussi bien à la naissance de nouveaux mythes qu'au développement d'anciens». Elle a mené ses recherches empiriques sous forme d'entretiens n'ayant pas le profil d'interviews. Elle soutient qu'avec un questionnaire préparé à l'avance, il n'aurait pas été possible de ressentir l'intensité, la profondeur et la sensibilité du phénomène ethnologique observé. Immédiatement après le dialogue avec les personnes, qu'elle connaissait personnellement, elle notait ses impressions à propos de ces entretiens.

\section{Anciens et nouveaux mythes}

Le premier exposé a été fait par le professeur d'études romanes Klaus Haitmann de l'université de Heidelberg. Il s'est intéressé à «L'analyse stylistique de la roumanité de Lucian Blaga ». Poète lyrique, dramaturge, philosophe, classique de la période moderne roumaine, L. Blaga (1895-1961) a créé une théorie mythologique sur la roumanité qui, jusqu'aujourd'hui, est restée la contribution la plus populaire à l'autognose roumaine. Son œuvre, Spatiul mioritic (l'espace mioritique), a été publiée en 1936. Le titre fait référence à la ballade populaire Miorita (l'agneau) et l'espace mioritique y est dépeint comme une contrée au relief peu accidenté, parsemée de villages roumains. L'auteur décrit l'âme roumaine qui imprègne les chants épiques populaires.

Blaga a créé une construction philosophique au moyen de laquelle il a expliqué l'essence de la roumanité, et a définit quelques déterminants :

44 (i) le sentiment de l'espace ;

(ii) le sentiment de destin;

(iii) le sentiment de communauté, déterminé au moyen de l'orthodoxie qu'il a comparée à un organisme vivant et divisée en de nouveaux déterminants par lesquels il explique les différences d'avec les religions catholique et protestante :

- la conscience de l'Église,

- la conscience de la nation,

- les formes de la langue,

- les différences de significations culturelles,

- les formes d'activités missionnaires,

- les représentations du salut,

- l'équipement de l'espace ecclésiastique, 
- les différentes formes de rituel ;

(iv) la conscience sophienne : en orthodoxie, la transcendance va de haut en bas (ex.: Sainte Sophie à Istanbul), tandis qu'en catholicisme, c'est l'inverse, de bas en haut (ex. : l'art gothique);

(v) l'orientation artistique vers des formes géométriques et linéaires ;

(vi) le penchant effréné pour des motifs pittoresques ;

(vii) le sens de la mesure et de la nuance.

L. Blaga, de par son œuvre controversée, a provoqué un intense débat encore actuel en Roumanie.

Le colloque a été clos par l'intervention du docteur Anton Sterbling de l'université militaire allemande d'Hambourg, "L'oppression, l'idéologie et le refoulement des mythes ». La fin du pouvoir communiste en Europe du sud-est n'a pas uniquement "réveillé l'histoire", elle a surtout revitalisé mythes et symboles nationaux tout juste disparus ou bien bouleversés dans leur sens. Sterbling s'est interrogé sur les circonstances spécifiques qui ont pu amener un réveil national qui s'est transformé, dans un nombre non négligeable de pays, en nationalisme ouvert.

Son hypothèse de départ est que les mythes nationaux ont subsisté aux interdictions et que ces interdits les ont nourris sans qu'il soit possible de parvenir à un dialogue constructif en ce qui les concerne. Les interdictions ont eu, par conséquent, un effet totalement négatif sur les mythes nationaux aussi bien dans les États multinationaux que dans les régions mononationales. Le principal effet de ces interdictions a été d'empêcher le dialogue avec ceux qui ne sont pas du même avis et les membres des autres nations. Bien qu'elles aient eu, durant une courte période, l'effet désiré, à long terme cependant, elles ont été plus que favorables au développement subculturel d'anciens mythes et à la naissance de nouveaux.

Le colloque de Bonn a montré qu'il y avait encore beaucoup à dire sur les mythes balkaniques. Les professeurs Holm Sundhausen et Wolfgang Höpken ont, hélas, annulé leur participation peu avant le début de cette réunion. Höpken devait parler du mythe de la guerre dans les Balkans, et Sundhausen avait annoncé qu'il évoquerait le mythe du Kosovo. Ainsi, le mythe le plus important d'un peuple jouissant d'une place centrale dans les Balkans aussi bien au niveau de la géographie que des courants historiques liés à cette position, n'aura pas été traité. Ce manque s'est fait sentir tout au long du colloque, surtout lors des débats. En dépit de cette lacune et de l'absence d'un spécialiste de la Yougoslavie, il convient de féliciter les organisateurs pour l'idée, l'organisation et l'agencement des exposés. Trois jours de travail consacrés aux mythes issus de toute l'Europe du sud-est ont, sans aucun doute, laissé une bonne impression sur tous ceux qui furent présents.

49 La remarque d'introduction du professeur Edgar Hösch, qui a présidé la première section, que toutes les nations, et tout particulièrement les petites, tendent vers la création de mythes, pourrait être révisée après cette réunion scientifique: que les grandes nations ont aidé les petites, avec un zèle considérable, à créer ou développer des mythes. On pourrait conclure qu'il y a encore beaucoup d'études à faire sur le développement des mythes balkaniques. Mais on pourrait également apporter des corrections à certaines versions historiques actuelles, en particuliers examiner l'influence de telle ou telle "grande puissance" sur la création et le développement des mythes chez les peuples de cet espace. 
50 Convaincus par le succès du colloque de septembre, les organisateurs ont l'intention de transformer ce rassemblement scientifique en une réunion périodique de spécialistes de l'Europe du Sud-Est qui se tiendrait tous les deux ans. 